EPJ Web of Conferences 19, 10004 (2012)

DOI: $10.1051 /$ epjconf/20121910004

(C) Owned by the authors, published by EDP Sciences, 2012

\title{
Radioactive and kinematic tracers of feedback from massive stars
}

\author{
R. Voss ${ }^{\mathrm{a}}$ \\ Department of Astrophysics/IMAPP, Radboud University Nijmegen, PO Box 9010, \\ 6500 GL Nijmegen, The Netherlands
}

\begin{abstract}
The mixing of ejecta from young stars into the interstellar medium is an important process in the interplay between star formation and galaxy evolution. A unique window into these processes is provided by the radioactive isotopes ${ }^{26} \mathrm{Al}$, traced by its $\gamma$-ray decay lines at $1.8 \mathrm{MeV}$. With a mean lifetime of $\sim 1 \mathrm{Myr}$ it is a long-term tracer of nucleosynthesis for massive stars. Our population synthesis code models the ejection of ${ }^{26} \mathrm{Al}$, together with the ${ }^{60} \mathrm{Fe}$, the kinetic energy and UV radiation for a population of massive stars. We have applied the code to study the nearby Orion region and the more massive Carina region and found good agreement with observational constraints.
\end{abstract}

\section{INTRODUCTION}

Feedback from massive stars plays a crucial role in the formation of stars, as it shapes the ISM and its subsequent star formation activity. The main feedback originates from the ejection of matter from massive stars through their winds and supernova explosions, and from their intense emission at short wavelengths into the UV. This UV radiation creates large photoionized regions around the stars, and the kinetic energy associated with ejection of stellar matter pushes at the ISM, together creating large shells and cavities.

We developed a new population synthesis tool to analyze groups of massive stars, where we model the emission of different forms of energy and matter from the stars of the association. In particular, the ejection of the two radioactive isotopes ${ }^{26} \mathrm{Al}$ and ${ }^{60} \mathrm{Fe}$ is followed, as well as the emission of hydrogen ionizing photons, and the kinetic energy of the stellar winds and supernova explosions. We investigate various alternative astrophysical inputs and the resulting output sensitivities, especially effects due to the inclusion of rotation in stellar models. As the aim of the code is the application to relatively small populations of massive stars, special care is taken to address their statistical properties. Our code incorporates both analytical statistical methods applicable to small populations, as well as extensive Monte Carlo simulations. A thorough description of the methods and the comparisons between different input models can be found in [1].

\section{MODELS OF THE ORION AND CARINA REGIONS}

We have used our population synthesis tool to analyze two regions with young massive stars, from which the ${ }^{26} \mathrm{Al}$ signal has been measured. The Orion OB1 association is located at a distance of $\sim 350-400 \mathrm{pc}$ and hosts several groups of young stars with ages 0-12 Myr. We have analyzed the stellar population in the Orion region and estimated that the total number of massive stars $\left(>8 M_{\odot}\right)$ formed there is $\sim 60$. The

\footnotetext{
ae-mail: rvoss@science.ru.nl
}

This is an Open Access article distributed under the terms of the Creative Commons Attribution-Noncommercial License 3.0, which permits unrestricted use, distribution, and reproduction in any noncommercial medium, provided the original work is properly cited. 

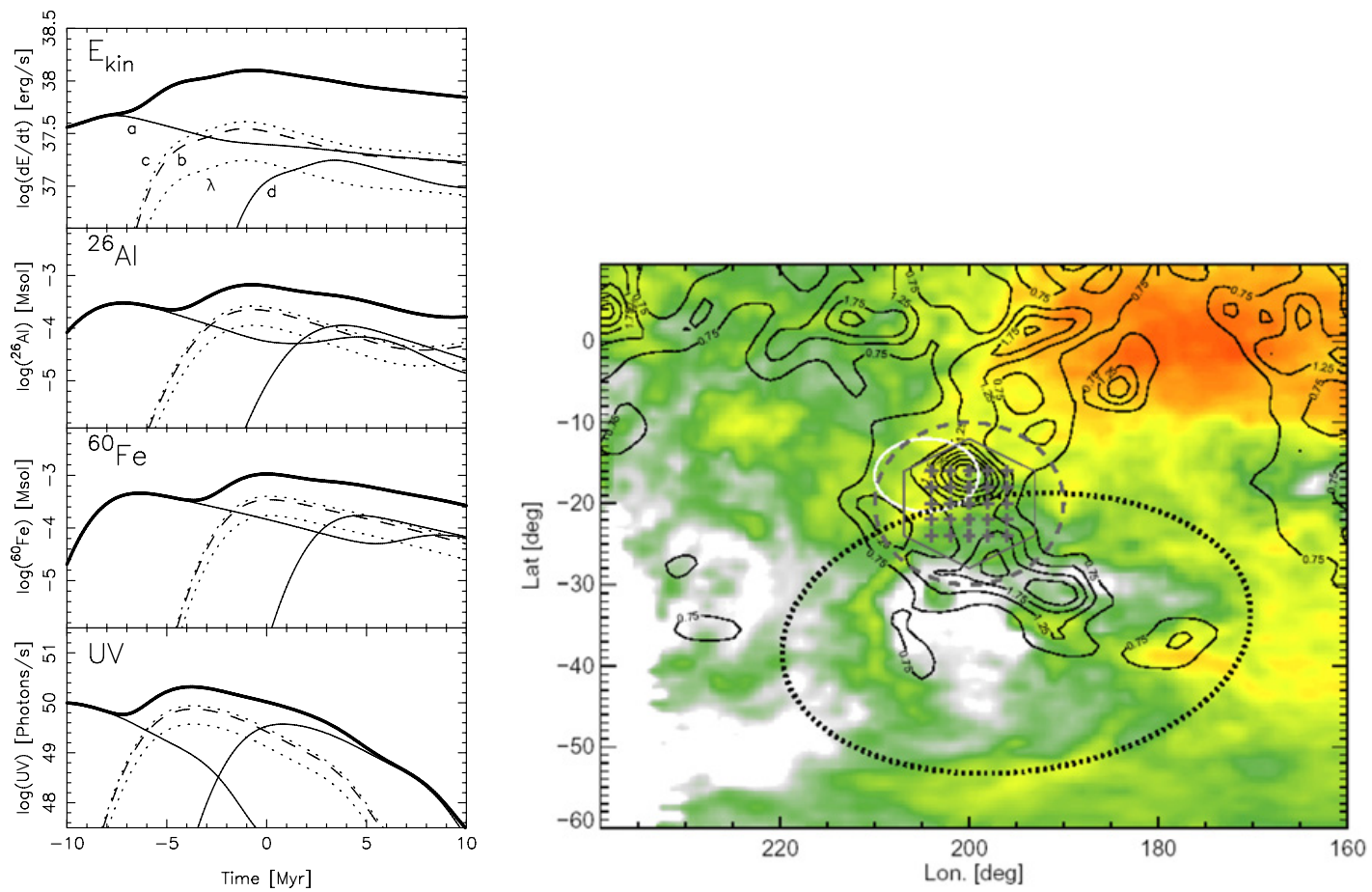

Figure 1. Left: Our simulated output of kinetic energy, ${ }^{26} \mathrm{Al},{ }^{60} \mathrm{Fe}$ and ionizing radiation from the massive stars in the Orion region. The thin lines indicates the outputs from the individual regions of the Orion OB1 association. It can be seen that the radioactive elements are good tracers of the kinetic energy output, making them useful for the study of feedback from massive stars. The results were shown to be consistent with observed properties of the region: ISM excavation by the kinetic energy, free-free emission from the ionized ISM and gamma-rays from the radioactive decay of ${ }^{26} \mathrm{Al}$. Right: The COMPTEL-observed $1.8 \mathrm{MeV}$ emission (contours), superimposed on a velocity integrated HI map of Orion-Eridanus bubble (color image). Orion OB1 is indicated (white ellipse), as well as the outer shell of the HI superbubble (large, black-dotted ellipse).

Carina region is located further away at a distance of $\sim 2.3 \mathrm{kpc}$, but hosts a larger population of young massive stars. We have estimated the total number to be $\sim 360$ stars $\left(>8 M_{\odot}\right)$. With our population synthesis tool we calculated the history of kinetic energy, ${ }^{26} \mathrm{Al}$ and ${ }^{60} \mathrm{Fe}$ and UV radiation output from the massive stars in Orion ([2]) and Carina ([3]). The results of applying our population synthesis model to the Orion OB1 association are shown in figure 1.

The results were shown to be consistent with observed properties of the region: ISM excavation by the kinetic energy, free-free emission from the ionized ISM and gamma-rays from the radioactive decay of ${ }^{26} \mathrm{Al}$. In particular, the results support massive stellar models with relatively strong wind mass-loss, and therefore provide evidence against a reduction of mass-loss rates beyond the revision of

\section{References}

[1] Voss, R., Diehl, R., Hartmann, D. H., et al., A\&A 504 (2009), 531-542

[2] Voss, R., Diehl, R., Vink, J. S., \& Hartmann, D. H., A\&A, 520 (2010), A51

[3] Voss, R., Martin, P., Diehl, R., Vink, J. S., Hartmann, D. H., Preibisch, T., A\&A, submitted

[4] Vink, J. S., de Koter, A., \& Lamers, H. J. G. L. M., A\&A (2000) 362, 295-309 